\title{
A LOOK INSIDE WHITE OLEDS
}

_ Peter Bobbert ${ }^{1}$, Reinder Coehoorn ${ }^{2,1}$ - DOI: 10.1051/epn/2013504

- ${ }^{1}$ Department of Applied Physics, Eindhoven University of Technology-Email: p.a.bobbert@tue.nl I ${ }^{2}$ Philips Research Eindhoven - Email: reinder.coehoorn@philips.com

White organic light-emitting diodes (OLEDs) for lighting are finding their way to the market. These OLEDs have organic layers only a few nanometres thick. Improving their efficiency and stability requires nanometre-precision information about where the light is emitted.

Reconstruction of the emission profile from the measured emission and molecular-scale modelling provide a unique nanoscale look inside white OLEDs. 


\section{A} lmost everyone nowadays is familiar with the high-quality OLED displays of recent generations of smart-phones. The breakthrough of OLED TVs seems a matter of time. Advantages of OLED displays over the ubiquitous liquid-crystal displays (LCDs) are evident. LCDs require a permanently emitting white back-light, illuminating an array of switchable liquid-crystal cell pixels with colour filters, which tune brightness and colour of the transmitted light. Hence, LCD technology is based on light absorption. In contrast, OLEDs actively emit light of the desired colour from pixels of organic semiconductors. This leads to higher contrast ratios ("black" is really black) and lower power consumption. OLEDs for lighting applications [1] are presently under development, not in the first place because of power efficiency - present-day commercial inorganic LEDs are still more efficient - but because they are unique in being ultrathin large-area light sources which can be flexible and transparent. Therefore, an OLED lamp needs no special luminaire: it is its own luminaire. And as luminaire losses are avoided, the efficiency at a system level is expected to be competitive over a wide range of applications. The required broad-spectrum white light could in principle be obtained from a Red-GreenBlue pixelated OLED display with all pixels switched on. However, the lateral structuring needed for this would be unnecessarily complicated and expensive. White-OLED research therefore focuses on vertical multilayer structures, pioneered in Japan [2].Light of different colours is emitted in different organic layers stacked on top of each other, together composing white light. It is important that a large fraction of the light actually leaves the structure and can be used for illumination. An excellent review of the efficiency of the light outcoupling from OLEDs was given two years ago in this journal by Brütting et al. [3]. Here, we focus on the light generation, and in particular on the question where in these structures emission of the different colours takes place. There is an intimate relation between this question and the question of outcoupling, since the position where emission takes place directly determines the light outcoupling efficiency.

\section{A hybrid white OLED}

In recent work together with the Institut für Angewandte Photophysik (IAPP) of the Technische Universität Dresden and Philips Research Aachen, our groups studied within the European FP7 project AEVIOM (Advanced Experimentally Validated Integrated OLED Model) the multilayer white-OLED stack shown in Fig. 1 [4]. The OLED has been fabricated at the IAPP by thermal evaporation in ultra-high vacuum of the organic materials displayed in this figure. The structure is ideal for a fundamental study, because most of the used materials have been well characterized in literature and all relevant processes can be addressed. The generation of the primary colours in this OLED is based on a hybrid principle, used extensively nowadays in commercially available white OLEDs. Green and red light are generated in layers of a host organic semiconductor doped by green and red phosphorescent dyes. The use of this dyedoping for light generation in OLEDs has been pioneered by researchers from the US [5]. A heavy metal atom in such dye molecules (in this case iridium) opens up - by its strong spin-orbit coupling - a radiative decay pathway for excitons (Coulombically bound electron-hole pairs) with a triplet spin configuration (parallel spins of electron and hole) next to singlet excitons (antiparallel spins). Hence, almost all excitons formed in such layers decay under the emission of a photon. Hybrid OLEDs avoid the use of blue phosphorescent dyes - of which the stability is still an issue - by using instead blue-emitting molecules without a heavy metal atom, at which only the singlet excitons decay radiatively (fluorescence). This compromises the internal quantum efficiency, because the triplet excitons - 75\% of all formed excitons - have no efficient radiative decay pathway and are thus wasted. Still, the power efficiency of today's commercial hybrid white OLEDs is already a factor of three to four higher than that of incandescent light bulbs ( $\sim 40-60 \mathrm{~lm} / \mathrm{W}$ vs. $\sim 15 \mathrm{~lm} / \mathrm{W})$, combined with operational lifetimes exceeding 10,000 hours.

Of crucial importance to the functioning of multilayer OLEDs is that excitons are generated at the right place

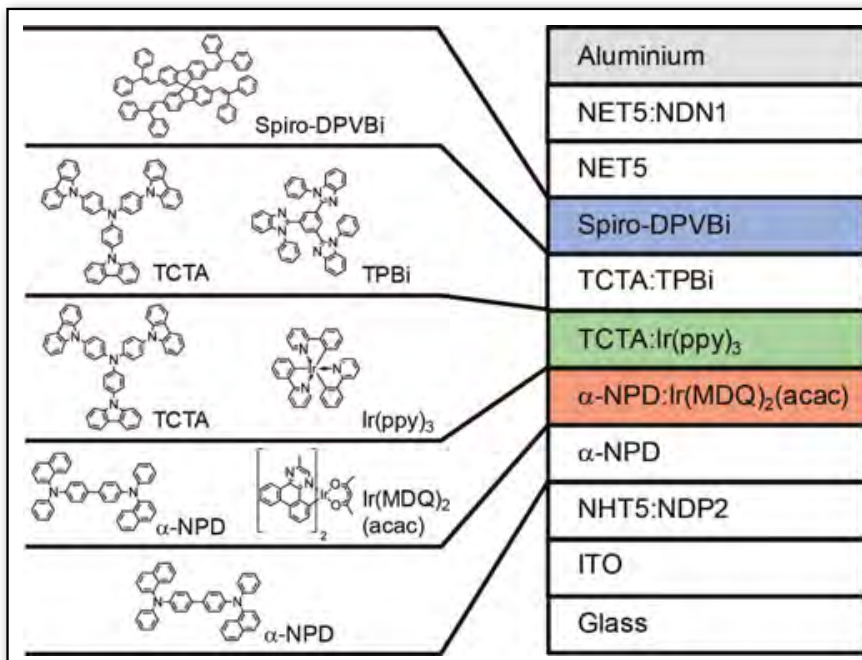

$\begin{aligned} 100 \mathrm{~nm} & \text { electron-injecting contact } \\ 40 \mathrm{~nm} & \text { n-doped electron-conducting layer } \\ 10 \mathrm{~nm} & \text { electron-transporting/hole-blocking layer } \\ 10 \mathrm{~nm} & \text { blue fluorescent layer } \\ 3 \mathrm{~nm} & \text { exciton-blocking interlayer } \\ 3 \mathrm{~nm} & \text { green phosphorescent layer } \\ 20 \mathrm{~nm} & \text { red phosphorescent layer } \\ 10 \mathrm{~nm} & \text { hole-transporting/electron-blocking layer } \\ 40 \mathrm{~nm} & \text { p-doped hole-conducting layer } \\ 90 \mathrm{~nm} & \text { transparent hole-injecting contact } \\ 1.5 \mathrm{~mm} & \text { substrate }\end{aligned}$


by encounter of electrons and holes, and that their subsequent motion until the moment of radiative decay is precisely controlled. In the OLED of Fig. 1 an excitonblocking interlayer has been inserted in between the blue and green layer. This interlayer prevents the motion of singlet excitons from the blue to the green layer as well as that of triplet excitons from the green to the blue layer. These are unwanted energetically downward processes that have to be blocked. On the other hand, motion of excitons from the green to the red layer can take place because of the direct contact between these layers. This is a desired process, because it leads to the right colour balance in this OLED, as will become clear.

Next to the control of the exciton motion, the control of the motion of electrons and holes is crucial. This control occurs by using organic semiconductors with appropriate energy levels of electrons and holes; see Fig. 2. First, electrons and holes have to be injected from suitable electrodes (of which at least one has to be transparent, in this case indium-tin-oxide, ITO) into the organic layers. In the early days of OLEDs, researchers struggled with the difference in energy between charges in the electrodes and in the organic semiconductor, leading to large barriers for charge injection. This problem has practically disappeared by an invention of the group at the IAPP. They introduced the use of highly $n$ - and $p$-doped organic layers adjacent to the electron- and hole-injecting contacts [6]. These layers behave as good electron and hole conductors, providing an almost barrier-free contact with the electrodes. From these doped layers the electrons and holes smoothly enter the electron- and hole-transporting layers, via which they move to the inner layers of the stack. The electron and hole energies of the organic semiconductors used in the transport layers are such that charge carriers of only one polarity can enter these layers. This guarantees that electrons and holes meet in the inner emissive layers of the stack and form excitons there. The OLED functions as an optical microcavity, in which exciton formation close to metallic electrodes must be avoided because this would lead to non-radiative decay. By carefully tuning the thicknesses of all layers, the outcoupling efficiency can be optimized [3]. A special role is again played by the interlayer between the green and blue layer. This very thin ( $3 \mathrm{~nm}$ ) layer should block excitons, but allow passage of both electrons and holes, in order to guarantee exciton formation in both the green and blue layer. In order to achieve this, the interlayer consists of a mixture of an electron transporter and a hole transporter, where the electron energy of the former matches well to that of the blue fluorescent material, while the latter material is the same as the hole-transporting host in the green layer.

\section{Reconstructed light-emission profile}

So, how does the OLED of Fig. 1 work? To answer this question, one would have to "look inside" the OLED, in order to find out where in the OLED the light is emitted.

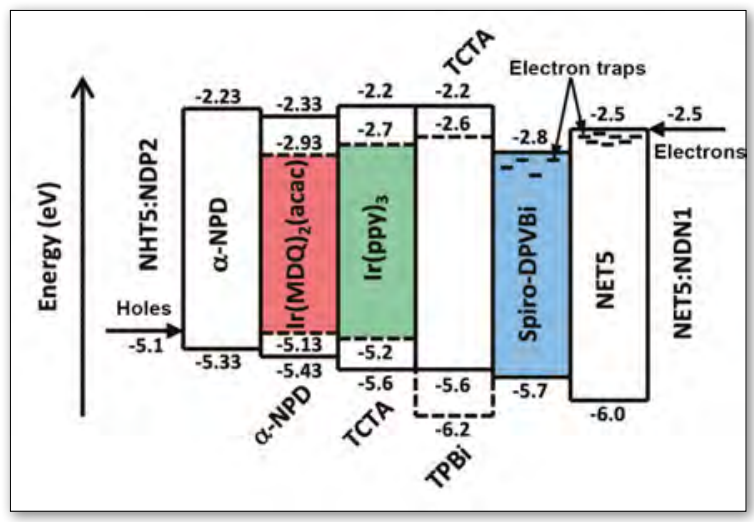

Here, the link with the light-outcoupling problem comes into play. If it is known where in the OLED the emission takes place, the external emission can be readily calculated by optical microcavity modelling, using the known dielectric properties of all layers in the stack and representing a radiatively decaying exciton as a classical dipole antenna [3]. The total outcoupled radiation of the OLED is the sum of the outcoupled radiation of all dipoles in the stack. However, it is also possible to solve the inverse problem, that is, to reconstruct the emission profile of all dipoles in the stack from knowledge of the total outcoupled radiation field [7]. Solving such an inverse problem is similar to reconstructing the three-dimensional (3D) inner structure of the earth from seismic signals (see the Feature by Jobert in this issue), or the 3D structure of a patient's body by $\mathrm{X}$-ray Computed Tomography (CT) scanning. Here, we make use of the strong dependence of the emission pattern on the distance of the dipole emitter to the aluminium cathode layer (see box). The information contained in the angle-, wavelength-, and polarization-dependent emitted light intensity is sufficiently rich to determine the dipoleemission profile of the primary colours with a nanometrescale precision. The result for the OLED of Fig. 1 at a typical operating bias voltage is given in Fig. 3(a). The balance between emission of the primary colours, with a strong red component, leads for this OLED to the emission of pleasant warm-white light. Resolution of the emission profile within the very thin $(3 \mathrm{~nm}$ ) green layer is just beyond the limits of the approach. The profiles in the red and blue layer are on the scale of a few nanometres confined to the interfaces with the green layer and interlayer, respectively.

\section{Simulations at the molecular scale}

Unique insight in the interplay of the processes leading to the emission profile can be obtained from molecularscale simulations. Whereas inorganic LEDs are based on crystalline semiconductors, in which the electronic and excitonic wave functions are delocalized, OLEDs are based on amorphous materials, in which the electronic contact between the molecules is weak. The quantummechanical wave functions of electrons, holes, and excitons in such disordered materials are localized on the molecules. Their motion involves sudden hops from one
$4 \mathrm{FIG}$. 2: Energy-level scheme of the OLED at open circuit. Indicated are the hole and electron energies of the highest occupied and lowest unoccupied molecular orbitals of the corresponding molecules, in eV. Due to disorder, these energies are actually broadened by approximately $0.1 \mathrm{eV}$. Electron traps are indicated in the two layers where they matter. 
molecule to another by an incoherent phonon-assisted tunnelling process. This motion can ideally be simulated by Monte Carlo methods, where at every time step a possible hop is chosen according to a randomly drawn number [8]. We have performed such simulations by modelling the OLED stack as an array of hopping sites representing all the different molecules in the stack, including the dyes [4]. Electron traps occur in many organic semiconductors. These are taken into account in the layers where they matter: the electron-transporting and blue fluorescent layers. The inter-site distance is taken to be $1 \mathrm{~nm}$, which is the typical distance between the molecules. All molecules are given an electron and a hole energy according to the energy-level scheme of Fig. 2, with a random energy due to disorder added. The doped electron- and hole-conducting layers are modelled as metallic-like contacts. The hopping rates are chosen to reproduce available experimental information about the mobility of electrons, holes, and excitons in each material in the stack. Coulomb interactions between all charges are taken into account. Electrons and holes attracting each other by the Coulomb force form excitons. Subsequent exciton motion is simulated within the green and red layer, and, importantly, from the green to the red layer. Excitons formed in the blue layer will stay there, because of the adjacent exciton-blocking interlayer. Information about the radiative decay efficiencies of the blue fluorescent and the green and red phosphorescent emitters, determining the fraction of excitons that decay by emitting a photon, is taken from experiments. This information is needed to predict the light-emission profile from the simulations. Excitons formed in the interlayer are assumed to be lost.

A first check of the validity of the simulations is the comparison between the calculated and measured current in the OLED. At the operating voltage of $3.6 \mathrm{~V}$ the current densities agree to within $25 \%$ [4], which is a gratifying result in view of the rather drastic approximations and assumptions made. In Fig. 3(b) the simulated exciton generation profile is given. We find that indeed almost all injected electrons and holes form excitons. As desired, most excitons form in the emissive layers, with the majority of excitons (54\%) formed in the blue layer. Also, a considerable fraction of excitons is formed in the

\section{BOX 1: RECONSTRUCTION OF THE LIGHT-EMISSION PROFILE}

The light-emission profile in OLEDs can be determined with nanometre-scale resolution, far below the diffraction limit [7]. That is possible due to the interference of directly emitted light from a dipole and light emitted from its image dipole in the highly conducting aluminium cathode layer. The left figure shows the angular dependence of the emission from a randomly oriented green emitter in glass, at varying distances from the cathode, for the two polarization directions. Due to interference, the perpendicular emission intensity oscillates as function of distance to the cathode with a periodicity $\lambda /(2 n) \cong 177 \mathrm{~nm}$, where $\lambda$ is the wavelength in vacuum (530 $\mathrm{nm}$ ) and $\mathrm{n}$ is the refractive index of glass (1.5). In the distance range from 80 to 160 $\mathrm{nm}$ (approximately from the first maximum for the perpendicular emission, near $\lambda /(4 n)$, to the first minimum, near $\lambda /(2 n)$ ) the emission profile depends for both polarizations strongly on the emission angle. Although the emission in actual OLEDs is influenced by the more complex layer structure, the dominant role of the metal cathode on the light emission pattern is preserved. In order to prevent loss of information due to internal reflection at the interface between the glass substrate and air, a glass hemisphere is used to extract also emission at large internal angles (figure on the right). Optical microcavity modelling and leastsquares fitting methods are used to obtain the emission profile in the OLED from the measured angle-, wavelength- and polarization-dependent emission.

$\checkmark$ LEFT: Polar diagrams of the normalized s and p-polarized emission from a randomly oriented dipole emitter in glass as a function of distance to an aluminium layer. RIGHT:Measurement configuration. A glass hemisphere is put on top of the 0 LED to extract also light which is internally emitted under angles larger than the critical angle for full internal reflection, $\theta_{\mathrm{cr}} \approx 42^{\circ}$

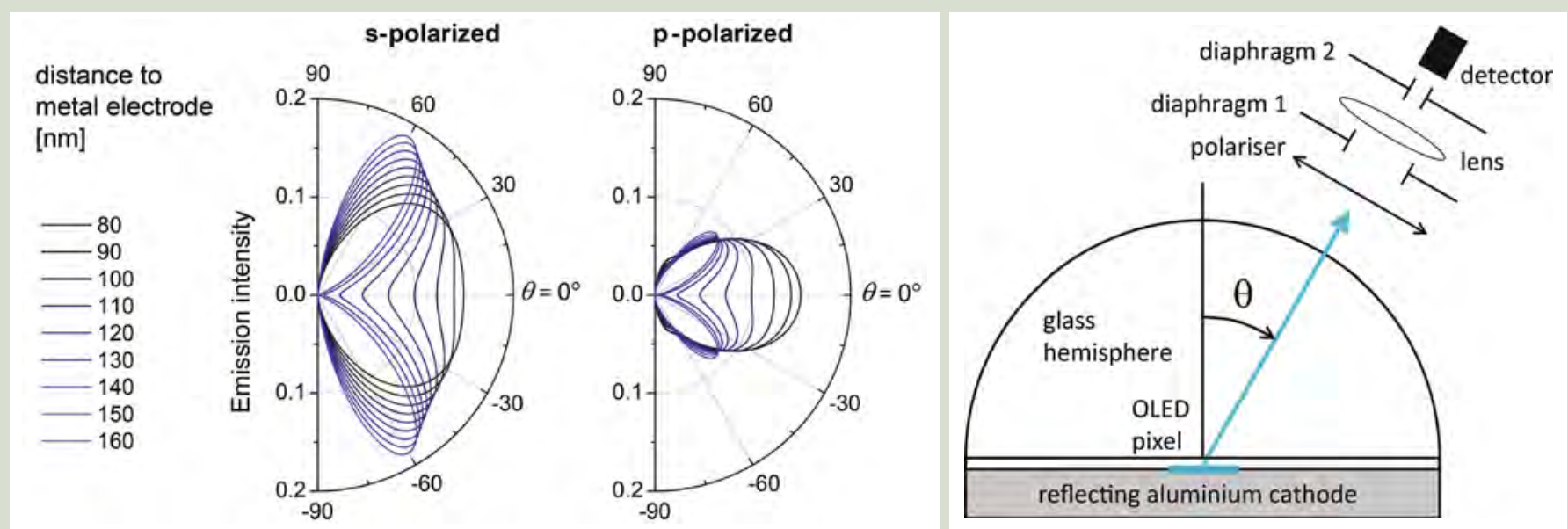


green layer (22\%), while almost no excitons are formed in the red layer (3\%). Fig. 3(b) reveals an important loss mechanism caused by excitons formed in the interlayer (21\%). This leads to a somewhat suboptimal efficiency, as is indeed observed.

After taking into account the excitonic motion and radiative decay efficiencies the simulated light-emission profile of Fig. 3(c) is obtained. We observe the same large component of emitted red light as found in the reconstruction of the experimental light-emission profile of Fig. 3(a), which is almost completely caused by transfer of excitons from the green to the red layer.Also the green and blue component of the simulated emission profile are in fair agreement with the reconstructed emission profile. Like the reconstructed profiles, the simulated profiles are confined to nanometre-scale regions close to the interfaces. The overall agreement between the reconstructed and simulated emission profiles is striking.

\section{A bright future}

The experimental and simulation tools discussed here provide a unique way to "look inside the OLED" and can be fruitfully used in the further development of white OLEDs, which show ever more complex layer structures. They could help in identifying what processes limit their efficiency and lifetime. An important current issue is to what extent interactions between two excitons and between excitons and charges lead to exciton quenching, and thereby to a loss of efficiency. It is found that at high voltages the efficiency of OLEDs drops (an effect called "roleoff"), which is presumably caused by these interactions. We are presently investigating this issue using our tools. With all-phosphorescent OLEDs, power efficiencies beyond $100 \mathrm{~lm} / \mathrm{W}$ have been demonstrated in laboratory experiments, even exceeding those of fluorescent lamps $(\sim 90 \mathrm{~lm} / \mathrm{W})$, while operating at a high luminance $[9,10]$. Novel concepts for efficient and stable organic light emitters are also emerging, such as organic emitters in which the energies of singlet and triplet excitons are almost identical [11]. These emitters allow a transformation of non-emissive triplet excitons into emissive singlet excitons, providing an alternative to phosphorescent emitters. Use of these emitters could lead to a radical redesign of OLEDs, to which our tools can also be applied. But whatever turn this will take, the future of OLEDs is bright.

\section{About the authors}

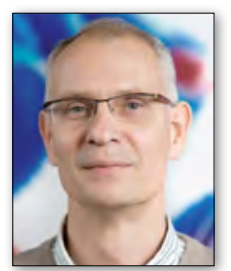

Peter Bobbert (1960) studied and obtained his $\mathrm{PhD}$ (cum laude) in Theoretical Physics in Leiden. After a postdoc in Delft as Royal Dutch Academy of Sciences Fellow he joined Eindhoven University in 1991. He presently works on various aspects of the theory of Organic Electronics, among which OLED modelling.

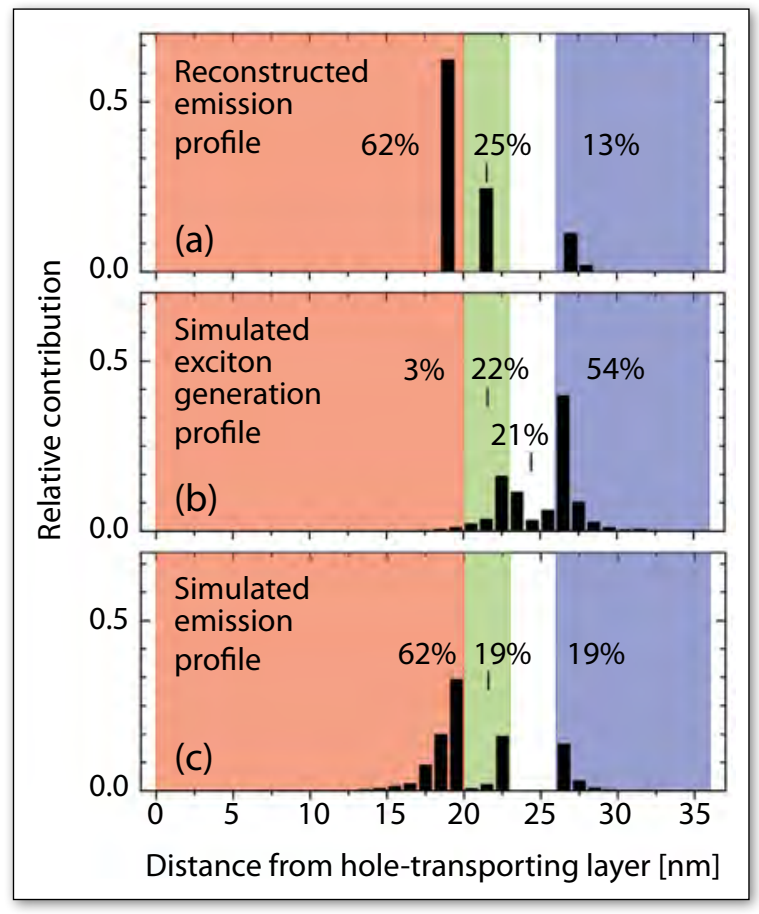

4 FIG. 3:

(a) Reconstructed light-emission profile of the OLED, at a bias voltage of $3.6 \mathrm{~V}$. (b) Simulated exciton generation profile. (c) Simulated lightemission profile. The difference between (b) and (c) is caused by motion of excitons from the green to the red layer and by radiative emission probabilities smaller than unity. Excitons generated in the interlayer (white) are lost.

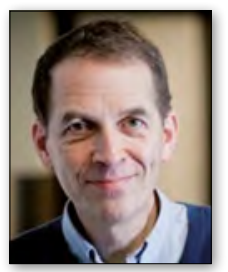

Reinder Coehoorn (1956) studied experimental physics in Amsterdam and obtained his $\mathrm{PhD}$ in Groningen. Since 1985, he is employed at Philips Research, working on permanent magnets, magnetic multilayers, and presently on OLEDs. He is part-time professor at the Eindhoven University, and an elected member of the Royal Netherlands Academy of Sciences.

\section{References}

[1] For example Philips: www.lighting.philips.com/main/led/oled/ and Osram:www.osram.com/osram_com/news-and-knowledge/oled-home

[2] J. Kido, M. Kimura, K. Nagai, Science 267, 1332 (1995)

[3] W. Brütting, J. Frischeisen, B.J. Scholz, T.D. Schmidt, Europhys. News 42, 20 (2011)

[4] M. Mesta, M. Carvelli, R.J. de Vries, H. van Eersel, J.J.M. van der Holst, M. Schober, M. Furno, B. Lüssem, K. Leo, P. Loebl, R. Coehoorn, P.A. Bobbert, Nature Mater. 12, 662 (2013)

[5] M.A. Baldo, D.F. O'Brien, Y. You, A. Shoustikov, S. Sibley, M.E. Thompson, S.R. Forrest, Nature 395, 151 (1998)

[6] K. Walzer, B. Maennig, M. Pfeiffer, K. Leo, Chem. Rev. 107, 1233 (2007)

[7] S.L.M. van Mensfoort, M. Carvelli, M. Megens, D. Wehenkel, M. Bartyzel, H. Greiner, R.A.J. Janssen, R. Coehoorn, Nature Photon. 4, 329 (2010); M. Carvelli, R.A.J. Janssen, R. Coehoorn, J. Appl. Phys. 110, 084512 (2011)

[8] For a movie, see www.youtube.com/watch?v=n1qkzv_ Q7UU\&feature $=$ youtu.be

[9] S. Reineke, F Lindner, G. Schwartz, N. Seidler, K. Walzer, B. Lüssem, K. Leo, Nature 459, 234 (2009)

[10] Y.-S. Park, S. Lee, K.-H. Kim, S.-Y. Kim, J.-H. Lee, and K.-J. Kim, Adv. Funct. Mat. (2013), DOI:10.1002/adfm.201300547 (advance online publication).

[11] H. Uoyama, K, Goushi, K. Shizu, H. Nomura, C. Adachi, Nature 492, 234 (2012) 